\title{
NOTCH EFFECT ON THE FRACTURE OF SEVERAL ROCKS: APPLICATION OF THE THEORY OF CRITICAL DISTANCES
}

\author{
J. Justo ${ }^{\mathrm{a}}$, J. Castro ${ }^{\mathrm{a} *}$, S. Cicero ${ }^{\mathrm{b}}$, M.A. Sánchez-Carro ${ }^{\mathrm{c}}$, R. Husillos ${ }^{\mathrm{d}}$ \\ ${ }^{a}$ Group of Geotechnical Engineering, Universidad de Cantabria, E.T.S de Ingenieros de Caminos, Canales y Puertos, \\ Av/ Los Castros 44, 39005, Santander, Spain \\ ${ }^{b}$ LADICIM (Laboratory of Materials Science and Engineering), Universidad de Cantabria, E.T.S de Ingenieros de \\ Caminos, Canales y Puertos, Av/ Los Castros 44, 39005, Santander, Spain \\ ${ }^{\mathrm{c}}$ Group of Engineering Geology. Universidad de Cantabria, E.T.S de Ingenieros de Caminos, Canales y Puertos, Av/ \\ Los Castros 44, 39005, Santander, Spain \\ ${ }^{\mathrm{d}}$ Group of $\mathrm{R}+\mathrm{D}+\mathrm{i}$ Cartographic Engineering and Mining, Escuela Politécnica de Ingeniería de Minas y Energía, \\ Boulevard Ronda Rufino Peón n 254. 39316 Torrelavega - Cantabria. \\ * Contact person: castrogj@unican.es
}

\begin{abstract}
The paper analyses the fracture behaviour of several rocks, namely a sandstone, a limestone and two marbles, one of them being a Carrara marble. The experimental program comprises in total 216 fracture specimens, tested in 4-point bending conditions and including specimens with notch radii varying from $0.15 \mathrm{~mm}$ up to 15 $\mathrm{mm}$. The notch effect is analysed through the evolution of the apparent fracture toughness and the application of the Theory of Critical Distances.
\end{abstract}

The present study aims to generalize a previous study on a granite and a limestone to a broader range of rocks. The point and line methods of the Theory of the Critical Distances successfully explain the notch effect on the fracture specimens. The value of the critical distance of these rocks is of the order of mm. Finally, the results show a correlation between the microstructural features of the rocks, specifically the grain size, and their critical distances.

KEYWORDS: Notch effect, Apparent fracture toughness, Theory of Critical Distances, marble, sandstone, limestone.

\section{INTRODUCTION}

It is well known that stress risers have a direct influence on the load-bearing capacity of structural components no matter what their nature: cracks, notches, holes, etc. Their presence plays a key role in the analysis of the corresponding structural integrity, and there are different approaches depending on the kind of stress riser that is studied. With regard to rocks, they cannot be considered a homogeneous continuum due to the small discontinuities like microcracks, pores, grain boundaries, etc. that accompany the rock matrix. These defects act as stress risers generating stress concentrations around them, leading to crack initiation and propagation and, eventually, to brittle failure. This problem can be of interest for several fields within civil (e.g., slopes, foundations), mining (e.g., tunnelling, drilling) and energy engineering (e.g., exploitation of geothermal energy). In addition to the above geometric aspects, stress concentration can also be caused by loading conditions such as bending and torsion, which tend to concentrate stresses at the surface leading to stress gradients. However, this paper will focus on the effect of geometric features, since loading stress concentration is generally negligible in comparison to the other aspects [1].

Microscopic defects are certainly likely to be found in geological materials. However, notches or defects with relatively large radii should not use the same expression as cracks to describe the stress field near the defect. Stress fields in the notch tip have already been modelled by numerous authors who generally observe a stress reduction acting perpendicular to the notch plane [2-5]. This stress reduction becomes more significant with the increase in the notch radius, which means that a given component will have a higher load-bearing capacity 
and apparent fracture toughness in notched conditions rather than in sharp cracks. However, the term 'sharp' depends on the analysed material, as there may be materials that behave as notches for very small notch radii [6] while others require relatively large radii to develop a notch effect [7]. It is therefore necessary to search for specific tools for the assessment of notch components, leaving aside the over-conservative application of the ordinary fracture mechanics that have commonly been used to deal with this problem. For this reason, the fracture analysis of rocks containing notch-type defects will be the main objective of this paper, aiming to apply a more accurate and less over-conservative approach than those assuming that all stress risers behave as crack-type defects.

\section{THEORETICAL BACKGROUND ON NOTCH FRACTURE AND THE THEORY OF CRITICAL DISTANCES}

The stress distribution normal to the notch plane $\left(\sigma_{y y}\right)$ has been represented by many authors over the last few decades [3,5,8-11]. Basically, they all suggest a distribution function with a form similar to $\sigma_{y y}=f(1 / \sqrt{r})$ but with certain differences, $r$ being the distance to the notch tip. If we represent the stress distribution at the notch tip in a bi-logarithmic form (Figure 1), we can distinguish three different regions $[4,12]$ : the first one is located at the closest zone to the notch tip (I) and corresponds to an almost constant stress zone, followed by a transition zone (II) that leads to region III, which can be considered as a zone of pseudo-singularity of stress, where the stress normal to the notch plane follows the next expression:

$$
\sigma_{y y}=\frac{K_{\rho}}{(2 \pi r)^{\alpha}}
$$

where $K_{\rho}$ is the notch stress intensity factor (NSIF) and $\alpha$ is a material constant for a given notch radius.

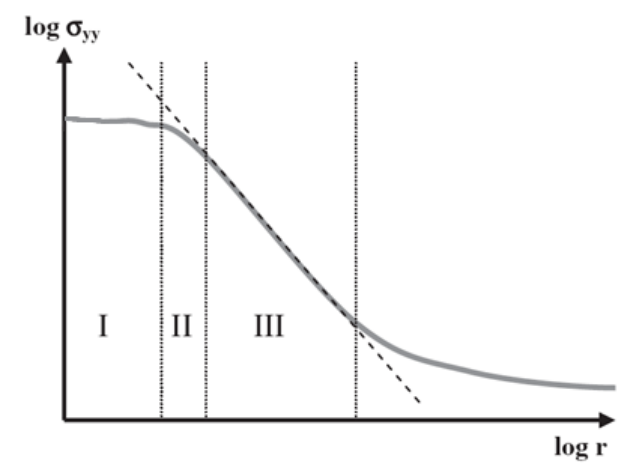

Figure 1. Bi-logarithmic representation of the stress distribution at a notch tip.

According to the notch theory, we can basically identify three brittle fracture criteria: global, local and nonlocal fracture criterion $[1,4,13]$. The first one is designed to predict fracture by considering the entire body (e.g., Linear Elastic Fracture Mechanics models) and states that the NSIF is limited by a certain critical value, $K_{\rho}^{c}$, which depends on the material and notch radius. When this critical value is reached, failure occurs $\left(K_{\rho}=\right.$ $K_{\rho}^{c}$ ), where $K_{\rho}$ defines the stress and strain fields next to the notch tip (see Eq. (1)), and $K_{\rho}^{c}$ represents the fracture toughness. It can be seen that this approach is analogous to the one proposed by linear-elastic fracture mechanics for the analysis of cracks $\left(K_{I}=K_{I C}\right)$, but limited by the lack of analytical solutions for $K_{\rho}$ and standardised procedures for the experimental definition of $K_{\rho}^{c}$.

With regard to local and non-local fracture criteria, they are both based on the stress-strain field at the notch tip and are relatively easy to implement in complex bodies. The main difference lies in the fact that local models use only data from a particular point for the calculations at that point, while non-local models also take information from other points that usually prescribe a surrounding volume. However, the distinction between local and non-local models is not always clear in practice, as in the case of the Theory of Critical Distances (TCD). 
The TCD can be considered a group of methods with certain common features: the use of a characteristic material length parameter (the critical distance, $L$ ) and the use of linear elastic analyses when performing fracture assessments $[1,14]$. It was first used by $[15,16]$, but it has been over the last few years, with the development of finite element stress analyses, that it has been scientifically studied and applied to different materials (ceramics, polymers, metals and composites). Despite its simplicity and potential for the analysis of fracture processes as demonstrated for a wide range of materials, scarce work can be found about the application of the TCD in rocks [17-19], it having started relatively recently to gain importance [20]. Geological materials should, in general, be suitable for the application of the TCD due to their mechanical properties. Their brittle behaviour, high inherent strength and low toughness make rocks highly sensitive to the presence of stress risers such as notches, cracks or any other defects. This simplifies to a great deal the performance of the experimental program from a scale point of view, since relatively large values of $L$ might be expected, of the order of few millimetres. The expression for the critical distance $L$ is as follows:

$$
L=\frac{1}{\pi}\left(\frac{K_{I C}}{\sigma_{0}}\right)^{2}
$$

where $K_{I C}$ is the material fracture toughness and $\sigma_{0}$ a characteristic material strength parameter (inherent strength), which, in the case of rocks, may be assumed to roughly coincide with the tensile strength $\sigma_{u}$, due to the linear-elastic behaviour at micro and macroscale [1].

All the methodologies included within the TCD are linear and allow the use of elastic continuum mechanics approaches. Some of the methods (Imaginary Crack Method, ICM; Finite Fracture Mechanics, FFM) are based on energy concepts for the assessment of a finite size crack propagation and thus use material parameters such as the critical strain energy release rate during crack growth, $G_{c}$, or the fracture toughness, $K_{c}$. However, other methods like the Point Method (PM) or the Line Method (LM) focus on the stress field at the defect tip, where they calculate a stress value and equate it to a characteristic strength of the material. All these methods consider the material inhomogeneity through a single parameter with length dimensions and offer quite similar predictions [1]. For this reason, those methods with far simpler and more straightforward application will be used in this work (PM and LM).

The Point Method (PM) is the simplest form of the TCD and states that failure will occur when stress at a certain distance $r_{c}$ reaches the inherent stress ( $\sigma_{u}$ for rocks). This method assumes linear-elastic behaviour of the material and can easily be demonstrated from both the stress field in the crack tip at failure defined by $[1,21]$, and from the definition of $L$ shown in Eq. (2):

$$
\frac{K_{I C}}{\sqrt{2 \pi r_{c}}}=\sigma_{0} \rightarrow r_{c}=\frac{1}{2 \pi}\left(\frac{K_{I C}}{\sigma_{0}}\right)^{2}=\frac{L}{2}
$$

Thus, the PM prediction can be written as $\sigma(L / 2)=\sigma_{0}$. The Line Method (LM) is based on the same principles as the PM, with the only difference that the stress parameter used is the average stress over a distance $d$ starting at the defect tip instead of the stress at a distance $r_{c}$ :

$$
\frac{1}{d} \int_{0}^{d} \sigma(r) d r=\sigma_{0}
$$

Once again, from the stress field at the crack tip and the definition of $L$ exposed in Eq. (2), we can analytically demonstrate that $d=2 L$ :

$$
\frac{1}{d} \int_{0}^{d} \frac{K_{I C}}{\sqrt{2 \pi r}} d r=\frac{2}{\sqrt{2 \pi}} \frac{K_{I C}}{d^{0.5}}=\sigma_{0} \rightarrow d=2 L
$$


Thus, the LM criterion can be written as:

$$
\frac{1}{2 L} \int_{0}^{2 L} \sigma(r) d r=\sigma_{0}
$$

Finally, an Area Method (AM) and a Volume Method (VM) could also be included within the TCD, which basically involve, respectively, an average stress over a certain area in the neighbourhood of the notch or over a volume. These methods have not been considered here, since fracture assessment becomes much more complicated and requires finite element analyses. By contrast, both PM and LM allow fracture assessment of components with any kind of stress risers in a simple way. In the case of the PM, it is enough to carry out two fracture test with specimens of the same material and geometry but with different type of defects (e.g., with different notch radii). The resulting stress-distance curves at fracture shown in Figure 2 can be obtained either by analytical solutions or by finite element analyses, and will theoretically cross each other at a distance $L / 2$ from the tip and with a stress equal to $\sigma_{0}$, as predicted by Eq. (3).

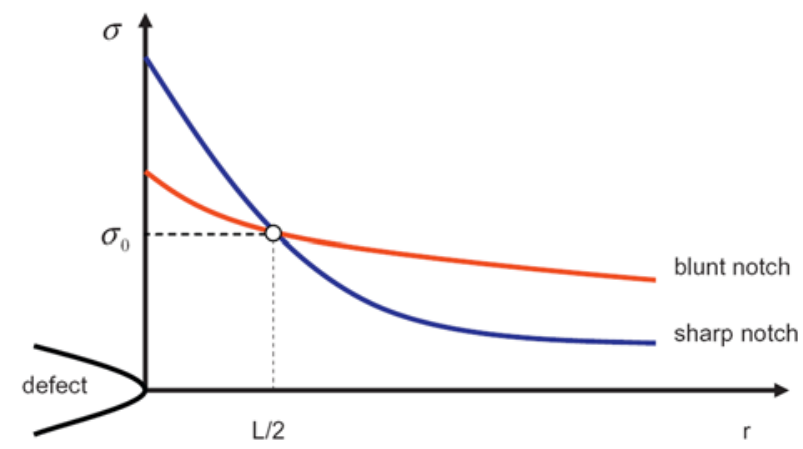

Figure 2. Stress distribution at a notch tip of blunt and sharp notches. Obtaining $L$ and $\sigma_{0}$ parameters.

To conclude, components containing U-shaped notches can easily be analysed by the TCD. One important reason for using these notched specimens is the simplicity they offer from a technical point of view to perform tests that enable fracture toughness, $K_{c}$, to be measured. Metallic materials do permit pre-cracking by fatigue loading for example, but introducing macroscopic cracks of controlled length into rocks is much more difficult due to their brittle condition which makes crack propagation unstable. The easiest way to introduce a cracklike defect without causing residual stresses is by means of a sharp slot machined into the specimen with a diamond wire for instance. Of course, this slot will have a finite radius, so we will obtain an apparent fracture toughness $\left(K_{I N}\right)$ different from the strictly true fracture toughness of the material, assuming that the notch is a sharp crack $(\rho=0)$. In essence, fracture analysis of U-shaped notch components is equated to a situation in a cracked component, where $K_{I N}$ is considered instead of $K_{I C}$ :

$K_{I}=K_{I N}$

Fracture will occur when Eq. (7) is fulfilled, where $K_{I}$ is the SIF for a crack with the same length as the notch. The PM and LM within the TCD provide expressions for the calculus of $K_{I N}$ based on the stress distribution of [2] as a function of distance, which is only valid for long and narrow notches:

$$
\sigma(r)=\frac{K_{I}}{\sqrt{2 \pi x}}\left(1+\frac{\rho}{2 x}\right)
$$

where $x$ represents the distance from a point halfway between the notch tip and its centre of radius. This expression can be rewritten in terms of the distance $(r)$ from the notch tip, keeping in mind that $x=r+\rho / 2$ :

$$
\sigma(r)=\frac{K_{I}}{\sqrt{\pi}}\left(\frac{2(r+\rho)}{(2 r+\rho)^{3 / 2}}\right)
$$


We can apply once again the PM by setting $\sigma(r)=\sigma_{0}$ and $r=L / 2$, which means that failure will occur and Eq. (7) will be fulfilled:

$$
K_{I N}=\sigma_{0} \sqrt{\pi}\left[\frac{\sqrt{(L+\rho)^{3}}}{L+2 \rho}\right]
$$

The apparent fracture toughness is equal to the real fracture toughness when $\rho=0$, so Eq. (10) can be rewritten as follows:

$K_{I N}=K_{I C} \frac{\left(1+\frac{\rho}{L}\right)^{3 / 2}}{\left(1+\frac{2 \rho}{L}\right)}$

The difference between the apparent fracture toughness and the real fracture toughness is a function of the root radius normalised by the critical distance. In the case of the LM, stress needs to be calculated as an average through a certain distance, from $r=0$ to $r=2 \mathrm{~L}$ as seen in Eq. (5). This requires integrating Eq. (9) in that domain, obtaining the following result:

$$
\sigma_{a v}=\frac{K_{I}}{2 L \sqrt{2 \pi}}\left(2 \sqrt{\frac{\rho}{2}+2 L}-\frac{\rho}{\sqrt{\frac{\rho}{2}+2 L}}\right)
$$

The analytical solution for the LM is once again obtained proceeding in the same way as for the PM:

$$
K_{I N}=K_{I C} \sqrt{\frac{\rho}{4 L}+1}
$$

According to [1], both Eq. (11) and Eq. (13) offer similar and reliable solutions as long as infinite body dimensions can be considered.

\section{ANALYSED MATERIALS}
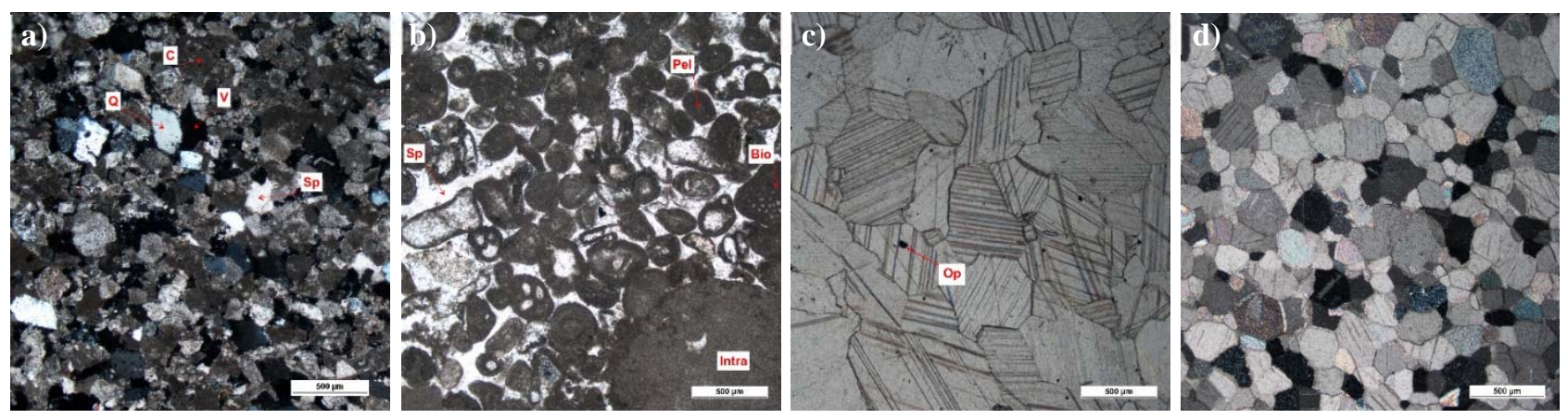

Figure 3. Analysed rocks: a) Floresta sandstone, Sedarenite (4x, crossed Nicol); b) Moleano limestone, Intrasparitic-pelsparitic or grainstone (4x, crossed Nicol); c) Macael marble (4x, parallel Nicol); d) Italian marble (4x, crossed Nicol).

\subsection{Floresta Sandstone (F) - classification: sedarenite}

$(F)$ is a sedarenite that consists of abundant carbonated grains and quartz (monocrystalline, polycrystalline and chert grains) (Figure 3a). Carbonates $[C]$ are mainly subrounded, medium size $(250-500 \mu \mathrm{m})$ and consist of 
intraclastic grains (mudstone texture), sparitic crystals and dolomite. Quartzes $[Q]$ are mainly subrounded and subangular shaped, angular occasionally, and sizes varies in the range of medium and very fine grain (62-500 $\mu \mathrm{m})$. Large packing and dissolution voids $[V]$ are common, cement $[S p]$ is very scarce and depositional structures are not recognizable.

\subsection{Moleano Limestone (C) - classification: intrasparitic - pelsparitic limestone [22] or grainstone [23]}

(C) is a limestone that consists of intraclasts, bioclasts, pellets and sparite crystals (Figure 3b). Intraclasts [Intra] show a micritic texture, are subrounded-rounded shaped and the size varies in the range of coarse sand and microconglomerate $(500-2000 \mu \mathrm{m})$. Bioclasts $[$ Bio $]$ are mainly fragments of bivalves and corals, which occasionally show internal sparitic recrystallization. Pellets $[\mathrm{Pel}]$ are the dominant allochemical component, size varies in the range of fine - medium sand $(125-500 \mu \mathrm{m})$ and always show a micritic internal texture. Sparite crystals $[S p]$ totally cement allochemical components.

\subsection{Macael (M) and Carrara/Italian (I) marbles}

Marbles show a very well developed granoblastic texture with equidimensional idiomorphs leucocratic crystals. Porosity is null (Table 1) in both of them and occasionally it is possible to identify opaque [Op] minerals in Macael (Figure 3c). The most important difference between the two types is crystal size. Italian marble crystals (Figure 3d) are smaller and better shorted $(112-500 \mu \mathrm{m})$ than in Macael $(200-1180 \mu \mathrm{m})$.

Table 1. Some technical properties of each material.

\begin{tabular}{lcccc}
\hline & $(\boldsymbol{F})$ & $(\boldsymbol{C})$ & $(\boldsymbol{M})$ & $(\mathbf{I})$ \\
\hline Bulk density $\left(\mathrm{kg} / \mathrm{m}^{3}\right)$ & 2320 & 2500 & 2715 & 2709 \\
Open porosity $(\%)$ & 16.3 & 6.4 & - & - \\
Water absorption $(\%)$ & 4.8 & 2.7 & 0.075 & 0.15 \\
Abrasion resistance $(\mathrm{mm})$ & 31 & 20 & 22.25 & 2.65 \\
\hline
\end{tabular}

\section{EXPERIMENTAL PROGRAM AND RESULTS}

\subsection{Splitting tensile strength tests}

A good characterisation of the tensile strength is crucial for the correct application of the TCD, since the inherent strength $\left(\sigma_{0}\right)$ is equivalent to the ultimate strength $\left(\sigma_{u}\right)$ in the case of rocks and brittle materials that do not experience plastification at the notch tip [1]. For this reason, twelve Brazilian tests were performed on each material following [24]. The results are presented in Table 2.

Table 2. Average tensile strength of each material.

\begin{tabular}{ccccc}
\hline & $(\boldsymbol{F})$ & $(\boldsymbol{C})$ & $(\boldsymbol{M})$ & $(\mathbf{I})$ \\
\hline Tensile strength $(\mathrm{MPa})$ & $3.38 \pm 0.40$ & $7.08 \pm 1.05$ & $8.15 \pm 1.61$ & $8.69 \pm 1.56$ \\
\hline
\end{tabular}

\subsection{Fracture toughness \& apparent fracture toughness}

At present, a wide range of testing methods can be found for fracture toughness evaluation in rocks with different specimen geometries (e.g. [25-27]). However, none of them has yet stood out enough to oust the other methodologies, and even those 'suggested methods for determining fracture toughness of rock' [26] specified by the International Society for Rock Mechanics (ISRM) have been frequently questioned or modified.

Among the several methodologies collected by [28] for fracture toughness assessment in rocks, [27] was selected for this work with some modifications based on [29]. This methodology was first proposed by [30] for ceramic materials, and makes use of single edge notch beam (SENB) specimens tested in 4-point bending, which ensures pure (without shear forces) and constant bending conditions among the inner loading points.

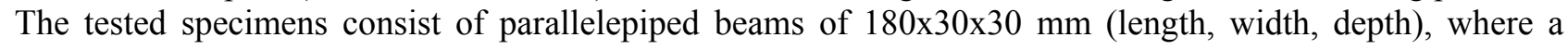
straight notch is cut in the middle to a depth of half-height (see Figure 4). Although the original method 
proposed by [30] establishes a height of $20 \mathrm{~mm}, 30 \mathrm{~mm}$ allows the tests to be performed with larger notch radii, while maintaining plane strain conditions. On the other hand, relative crack length $\left(\alpha_{0}\right)$, which is defined as the ratio between the initial crack length $\left(a_{0}\right)$ and the total height of the specimen $(h)$, has been limited to $0,45 \leq \alpha_{0} \leq 0,55$ in order to guarantee a high confinement.

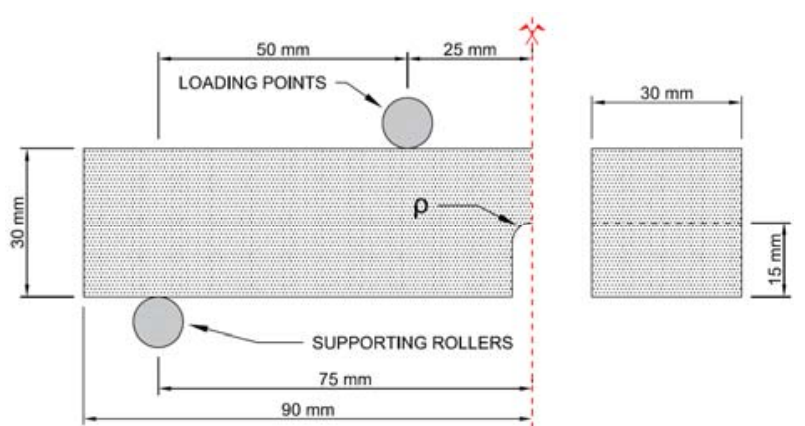

Figure 4. Scheme of fracture specimens. Notch radii ( $\rho)$ vary from $0.15 \mathrm{~mm}$ up to $15 \mathrm{~mm}$.

Despite the simplicity of the bending tests, it should be noted that the rollers are to be designed in such a way that they minimise friction between them and the specimens, and reduce possible torsion effects that may arise when opposite faces are not perfectly parallel. For this reason, the rollers will allow rotation as well as lateral tilting with the aim of improving their support conditions. In addition, the upper head of the device has been provided with a spherical joint that allows a greater degree of movement and adjustment, while ensuring that the axis of load application always remains centered.

Finally, fracture toughness values $\left(K_{I C}\right)$ for SENB specimens can be calculated with the following formulation initially developed and defined by $[27,30]$ :

$$
K_{I C}=\frac{F \cdot Y}{b \cdot h^{1 / 2}}
$$

where $b$ is the specimen depth, $h$ is the specimen height, $F$ is the failure load that can be easily determined with the applied load and vertical displacement records obtained from the 4-point bending tests, and $Y$ is the compliance factor given by the following expression:

$$
Y=\frac{3 \cdot\left(L_{0}-L_{i}\right) \cdot \alpha_{0}^{1 / 2} \cdot X}{2 h \cdot\left(1-\alpha_{0}\right)^{3 / 2}}
$$

with

$X=1.9887-\left[\frac{\left(3.49-0.68 \alpha_{0}-1.35 \alpha_{0}^{2}\right) \cdot \alpha_{0} \cdot\left(1-\alpha_{0}\right)}{\left(1+\alpha_{0}\right)^{2}}\right]-1.32 \alpha_{0}$

where $L_{0}$ and $L_{i}$ are respectively the spans between the supporting rollers and the inner loading points as depicted in Figure 4 (in this case, following standards for 4-point bending tests [29], $L_{0}=150 \mathrm{~mm}$ and $L_{i}=50$ $\mathrm{mm})$, and $\alpha_{0}$ is the above mentioned relative crack length $\left(\alpha_{0}=a_{0} / h\right)$.

With all this, six tests have been performed with this configuration for each material and notch radius, with a constant loading rate of $0.05 \mathrm{~mm} / \mathrm{min}$ till failure, which is consistent with the limit loading rate of $0.25 \pm 0.05$ $\mathrm{MPa} / \mathrm{s}$ established at [29]. Basically, cracked specimen formulation is being applied to notched specimens and, therefore, apparent fracture toughness $\left(K_{I N}\right)$ values and not real fracture toughness will be obtained. This will allow the limit where notch effect can no longer be negligible to be found. 
Table 3. Results of $K_{I N}$ and failure load for $(F),(C)$, (M) and (I).

\begin{tabular}{|c|c|c|c|c|c|c|c|c|c|c|c|}
\hline (F) & 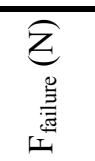 & 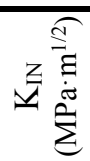 & (C) & 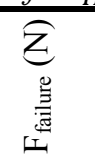 & 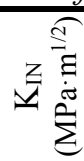 & (M) & 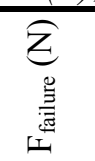 & 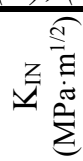 & (I) & 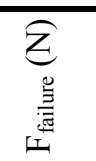 & 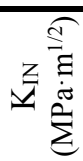 \\
\hline F-015-T1-1 & 193.8 & 0.42 & C-015-T1-1 & 377.7 & 0.70 & M-015-T1-1 & 610.8 & 1.19 & I-015-T1-1 & 302.1 & 0.67 \\
\hline F-015-T1-2 & 175.2 & 0.37 & C-015-T1-2 & 319.1 & 0.64 & M-015-T1-2 & 429.7 & 0.94 & & 418 & 0.87 \\
\hline F-015-T1-3 & 99.4 & 0.28 & $-015-\mathrm{T} 1-3$ & 485.7 & 0.94 & M-015-T1-3 & 577.3 & 1.19 & & 259.5 & 0.56 \\
\hline F-015-T1-4 & 137.4 & 0.32 & $015-\mathrm{T} 1-4$ & 303.2 & 0.65 & M-015-T1-4 & 654.4 & 1.30 & & 332.4 & 0.68 \\
\hline F-015-T1-5 & 264.5 & 0.43 & $-015-\mathrm{T} 1-5$ & 337.4 & 0.68 & M-015-T1-5 & 518.9 & 1.17 & I-015-T1-5 & 406.2 & 0.77 \\
\hline F-015-T1-6 & 180.7 & 0.39 & 015-T1-6 & 355.3 & 0.78 & M-015-T & 521.7 & 1.03 & I-015-T1-6 & 402.4 & 0.90 \\
\hline F-05-T1-1 & 188.9 & .37 & $-05-\mathrm{T} 1-1$ & 340.6 & 0.86 & M-05-T1-1 & 542 & 1.11 & I-05-T1-1 & 245.8 & 0.50 \\
\hline F-05-T1-2 & 172.7 & .34 & & 286.7 & 0.73 & M-05-T1-2 & 551.6 & 1.10 & & 431.9 & 0.99 \\
\hline F-05-T1-3 & 143 & 0.28 & $05-\mathrm{T} 1-3$ & 412.7 & 0.84 & M-05-T1-3 & 649.8 & 1.27 & $05-\mathrm{T} 1-3$ & 358.3 & 0.71 \\
\hline F-05-T1-4 & 188.1 & 36 & & 315.3 & 0.80 & M-05-T1-4 & 504.2 & 1.01 & & 413.2 & 0.86 \\
\hline F-05-T1-5 & 177.3 & 0.34 & $05-\mathrm{T} 1-5$ & 366 & 0.76 & M-05-T1-5 & 604 & 1.22 & $05-\mathrm{T} 1-5$ & 318.3 & 0.64 \\
\hline F-05-T1-6 & 192.5 & .40 & C-05-T1-6 & 358.9 & 0.73 & T1-6 & 608.4 & 1.25 & & 301 & 0.74 \\
\hline F-1-T1-1 & 133.7 & .28 & T1-1 & 363 & 0.78 & M-1 & 406.9 & 0.87 & $-\mathrm{T} 1-1$ & 353.2 & 0.76 \\
\hline F-1-T1-2 & 135 & 28 & & 421.9 & 0.90 & & 559.5 & .22 & & 276.5 & 0.61 \\
\hline F-1-T1-3 & 158.9 & 36 & $1-\mathrm{T} 1-3$ & 430.5 & 0.89 & M-1-T1-3 & 528.5 & 1.30 & -T1-3 & 271.8 & 0.59 \\
\hline F-1-T1-4 & 38.5 & 35 & & 341.6 & 0.77 & $1-4$ & 566.9 & .21 & & 26.2 & 0.87 \\
\hline F-1-T1-5 & 161.4 & .40 & $1-\mathrm{T} 1-5$ & 431.3 & 0.91 & I-1-T1-5 & 539.8 & 1.31 & $-\mathrm{T} 1-5$ & 327.6 & 0.68 \\
\hline & & 41 & & 478.3 & 1.01 & -6 & & 28 & & & .71 \\
\hline F-2-T1-1 & 161.7 & 37 & $2-\mathrm{T} 1-1$ & 398.6 & 0.88 & M-2-T1-1 & 605.9 & 1.24 & 2-T1-1 & 351.4 & 0.83 \\
\hline & 69.3 & & & 318 & 0.70 & & & 43 & & & .78 \\
\hline F-2-T1-3 & 215.9 & .45 & $-2-\mathrm{T} 1-3$ & 400.2 & 0.90 & M-2-T1-3 & 455.6 & 04 & 2-T1-3 & 382.4 & 0.92 \\
\hline & & & & & & & & & & & .64 \\
\hline F-2-T1-5 & 179.1 & .39 & $-2-\mathrm{T} 1-5$ & 415.5 & 0.93 & M-2- & 297.4 & 0.68 & $\mathrm{~T} 1-5$ & 374.5 & 0.90 \\
\hline & & & & & & & & & & & .70 \\
\hline F-4-T1-1 & 137.2 & .32 & $4-\mathrm{T} 1-1$ & 382.4 & 0.85 & $1-1$ & 601.5 & 1.66 & T1-1 & 406.2 & 0.95 \\
\hline & & & & & & & & & & & .65 \\
\hline F-4-T1-3 & 81.7 & 0.43 & $-4-T 1-3$ & 388.2 & 0.86 & $1-3$ & 677 & 0.94 & T1-3 & 302.4 & 0.70 \\
\hline & & & & & & & & & & & .62 \\
\hline F-4-T1-5 & 188.1 & & & 420 & 0.93 & -5 & 771.1 & .79 & $1-5$ & 302.3 & 0.71 \\
\hline F-4-T1-6 & & & & 4.8 & & M-4- & & 00 & T1-6 & 359.8 & .86 \\
\hline $4-\mathrm{T} 1-1 \mathrm{C}$ & 205 & 40 & $\mathrm{~T} 1-1 \mathrm{C}$ & 524.5 & 1.01 & $-4-T 1-1 \mathrm{C}$ & 589.7 & 1.17 & $\Gamma 1-1 \mathrm{C}$ & 261.2 & 0.54 \\
\hline F-4-T1-2C & & & -T1-2C & & & $-4-T 1-2 C$ & & & $11-2 C$ & & .70 \\
\hline F-4-T1-3C & 203 & .40 & $4-\mathrm{T} 1-3 \mathrm{C}$ & 591.4 & 1.16 & $1-4-T 1-3 C$ & 730 & 1.31 & $\Gamma 1-3 \mathrm{C}$ & 314.2 & 0.61 \\
\hline F-4-T1-4C & 213.2 & & & & & [-4-T1-4C & 939.4 & & $11-4 c$ & 323 & .64 \\
\hline F-4-T1-5C & 49.9 & 29 & $\mathrm{~T} 1-5 \mathrm{C}$ & 374.7 & 0.72 & $5 \mathrm{C}$ & 739.4 & 1.44 & $11-5 \mathrm{C}$ & 316.6 & 0.62 \\
\hline F-4-T1-6C & 08.6 & & 4-T1-6C & 386.8 & & M-4-T1-6C & 793.7 & 26 & T1-6C & 267.8 & 0.53 \\
\hline F-7-T1-1 & 23.8 & 44 & $7-\mathrm{T} 1-1$ & 446.2 & 0.92 & M-7-T1-1 & 589.6 & 1.15 & I-7-T1-1 & 374.5 & 0.74 \\
\hline F-7-T1-2 & +1.5 & & & 500.3 & & -2 & 700 & & T1-2 & 309.4 & 0.62 \\
\hline F-7-T1-3 & 112 & 42 & $-7-T 1-3$ & 523 & 1.04 & M-7-T1-3 & 730 & 1.42 & I-7-T1-3 & 298.2 & 0.59 \\
\hline F-7-T1-4 & 37.7 & & & & & M-7- & 939.4 & & -7-T1-4 & 299.2 & 0.59 \\
\hline F-7-T1-5 & 141 & & $-7-T 1-5$ & & & & 739.4 & 1.37 & $-7-T 1-5$ & 422 & 0.85 \\
\hline F-7-T1-6 & 46.6 & & $7-\mathrm{T} 1-6$ & 12.5 & & $1-6$ & 793.7 & & $-\mathrm{T} 1-6$ & 425.5 & 0.84 \\
\hline F-10-T1-1 & 015 & & & & & M-10-T1-1 & 801.9 & 1.54 & $-10-\mathrm{T} 1-1$ & 488.7 & 0.98 \\
\hline F-10-T1-2 & 190.4 & & 10-T1-2 & & & M-10-T1-2 & 716.5 & 1.43 & I-10-T1-2 & 84.2 & 0.77 \\
\hline F-10-T1-3 & 214 & & $-10-\mathrm{T} 1-3$ & 436.2 & 0.89 & M-10-T1-3 & 719.3 & 1.45 & I-10-T1-3 & 308.7 & 0.61 \\
\hline F-10-T1-4 & 226.2 & & & & & M-10-T1-4 & & 1.40 & & 346.8 & 0.70 \\
\hline F-10-T1-5 & 200.6 & & & & & & & 1.61 & & 339.8 & 0.68 \\
\hline & 220.9 & & & 450.3 & & & 692.2 & 1.35 & & 340.1 & 0.68 \\
\hline F-15-T1-1 & 211.1 & & C-15-T1-1 & & 0.80 & M-15-T1-1 & & 0.99 & & 410 & 0.83 \\
\hline F-15-T1-2 & 225.5 & & C-15-T1-2 & & 0.84 & M-15-T1-2 & 821.3 & 1.65 & & 405.2 & 0.85 \\
\hline F-15-T1-3 & & & & 383.5 & 0.80 & & & & & 391.3 & 0.79 \\
\hline & & & & & & & 500.7 & 1.00 & I-15-T1-4 & 349.6 & 0.69 \\
\hline F-15-T1-5 & 201.5 & & C-15-T1-5 & 391.1 & 0.81 & M-15-T1-5 & 863.9 & 1.73 & & 391.4 & 0.79 \\
\hline F-15-T1-6 & 193.9 & 0.38 & C-15-T1-6 & 525.6 & 1.10 & M-15-T1-6 & 820.1 & 1.65 & I-15-T1-6 & 281.7 & 0.55 \\
\hline
\end{tabular}


Table 3 gathers the results of $K_{I N}$ for the four analysed rocks with notch radii of $0.15 \mathrm{~mm}, 0.5 \mathrm{~mm}, 1 \mathrm{~mm}, 2$ $\mathrm{mm}, 4 \mathrm{~mm}, 7 \mathrm{~mm}, 10 \mathrm{~mm}$ and $15 \mathrm{~mm}$. Those notches with $\rho=0.15 \mathrm{~mm}$ can be assumed to behave as cracks providing a reasonable approximation to the real fracture toughness despite their finite radius. It can be observed how Macael Marble $(M)$ develops the highest values of load-bearing capacity and fracture toughness, while Floresta Sandstone $(F)$ shows the smallest values (but with less dispersion) as a consequence of the scarce cement between particles and homogeneity in grain size. Rocks are natural materials with large variability, and consequently there is an important scatter in the results.

a)

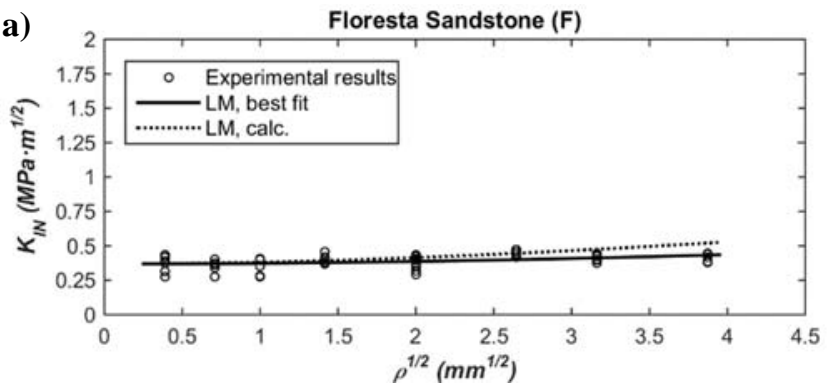

c)

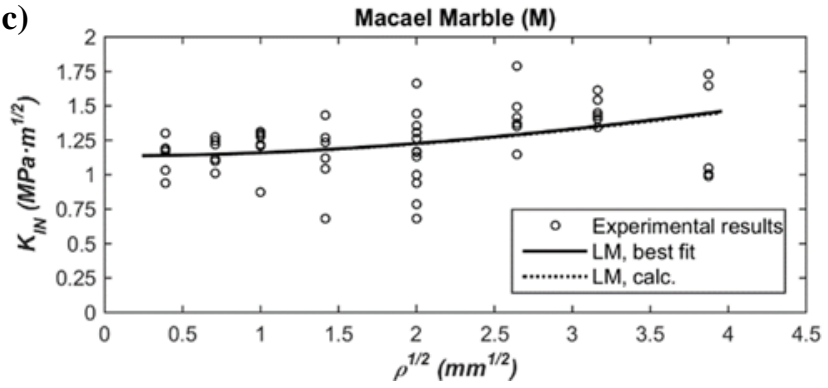

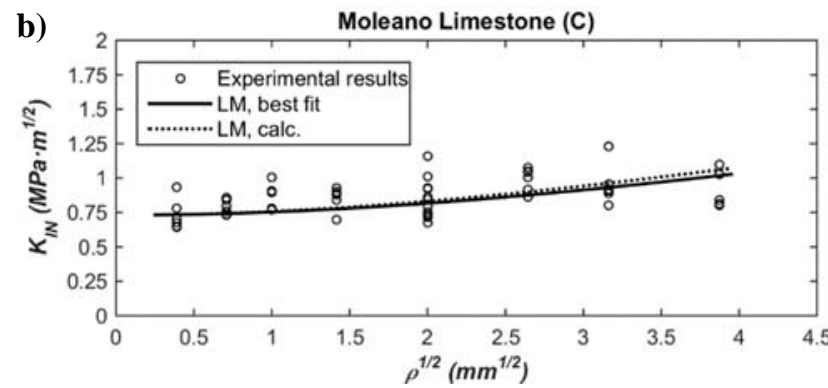

d)

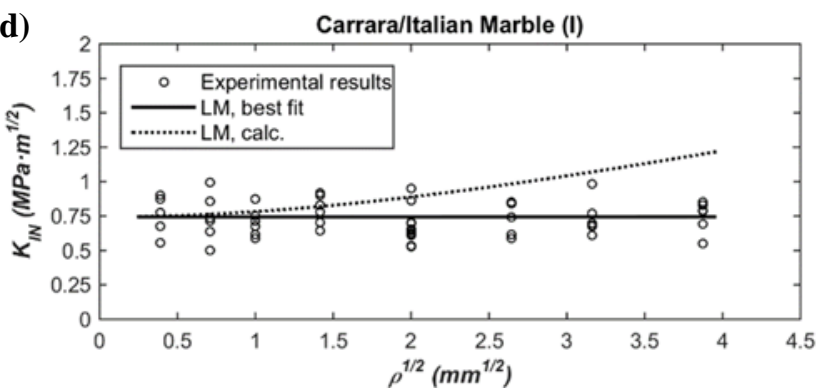

Figure 5. Apparent fracture toughness test results and comparison with the TCD (LM) for each material.

Figure 5 presents the experimental results for each material and establishes the best-fit curves (solid lines) and calculated curves (dotted lines) in each case, according to Eq. (13), which corresponds to the LM of the TCD. The calculated curves consider the averaged apparent fracture toughness values for $\rho=0.15 \mathrm{~mm}$ (Eq. (14)) and the resulting critical distance $L$ from Eq. (2), while best-fit curves are obtained by fixing the value of the apparent fracture toughness to the calculated one and leaving the critical distance $L$ as the only free variable. With all this, Table 4 includes the results of the calculated $L$ and $K_{I C}$, as well as the values of $L$ for best-fit curves providing least squares. The predictions provided by the PM have also been included in Table 4.

Table 4. Summary of the obtained results for $L$ and $K_{I C}$.

\begin{tabular}{lrrrc}
\hline & $(\boldsymbol{F})$ & $(\boldsymbol{C})$ & $(\boldsymbol{M})$ & $(\mathbf{I})$ \\
\hline Calculated $K_{I C}\left(\mathrm{MPam}^{1 / 2}\right)$ & 0.37 & 0.73 & 1.14 & 0.74 \\
Calculated $L(\mathrm{~mm})$ & 3.79 & 3.39 & 6.19 & 2.33 \\
\hline Best fit of $L(\mathrm{~mm})-$ LM (fixed $\left.K_{I C}\right)$ & 9.95 & 3.99 & 6.01 & - \\
\hline Best fit of $L(\mathrm{~mm})-$ PM (fixed $\left.K_{I C}\right)$ & 3.66 & 2.30 & 2.85 & 8.81 \\
\hline
\end{tabular}

According to [31], when $\rho / L<1$, the corresponding notch behaves as a crack of the same length, which means that notches whose radii are smaller than the obtained $L$ can be analysed by using ordinary fracture mechanics without considering notch effects and, therefore, $K_{I N}=K_{I C}$. In the case of rocks, $L$ is of the order of mm, so notches with significant root radii may behave as cracks. In Floresta Sandstone $(F)$, for example, it can be observed that the notch effect is almost negligible up to $\rho \approx 4 \mathrm{~mm}$ (Figure 5a), which agrees well with the expression of the normalised notch radius $(\rho / L<1)$. Good agreement between TCD predictions and laboratory results is obtained in all the cases except in the Carrara Marble (Figure 5d), where no tendency can be seen. This means that the notch effect is not appreciable for the considered notch radii as a consequence of the large value of $L$, so larger radii should be tested in order to appreciate that effect. Besides, noticeable differences were seen in the experimental results depending on the block of Carrara Marble $(I)$ from which the specimens were extracted, which introduces an important source of variability into the results. 
The adjustment between curves becomes less accurate as the notch radius gets larger (see Figures 5a, 5b). This is because the range of application of Eq. (13) that corresponds to the LM is limited by the stress distribution of [2], which is theoretically only valid for long and narrow notches. However, the predictions provided have proved to be reasonable even when this hypothesis is not totally fulfilled, as in the case of Figure 5c. This inaccuracy is the main factor responsible for the differences between the calculated and best-fit values of $L$ (see Table 4), although consequences due to variations in $L$ are rather limited since this variable is squared in Eq. (13).

Finally, the physical meaning of $L$ seems to be related to the corresponding material microstructure [1]. In the case of rocks, this correlation is thought to be somehow influenced by the grain size, which plays an important role in crack growth acting as an obstacle for propagation. Figure 6 shows, for each material, the range of values of $L$ (dashed lines) between the one corresponding to the best-fit of the LM (empty symbols) and that calculated by Eq. (2) (filled symbols) Both axes are in millimetres and are represented in a logarithmic scale. It seems to indicate the existence of some kind of relationship between both variables, although this is not totally clear yet. According to [32], the value of $L$ of a certain material is usually on a range between the mean grain size $D$ and ten times this value. This approximation is reasonably fulfilled in most of the analysed rocks. The calculated values of $L$ (Eq. (2)) for the Granite and the Carrara Marble are also within the mentioned range, although the values obtained by the best-fit curves (LM) of the experimental results are considerably far from the expected range in these two cases. In this sense, not only mean grain size but some other aspects like grain size density function, porosity and grain composition are likely to affect the resulting critical distance. Consequently, all these variables (especially the grain size density function) will be analysed in future research, in order to determine their influence on the corresponding value of critical distance $L$.

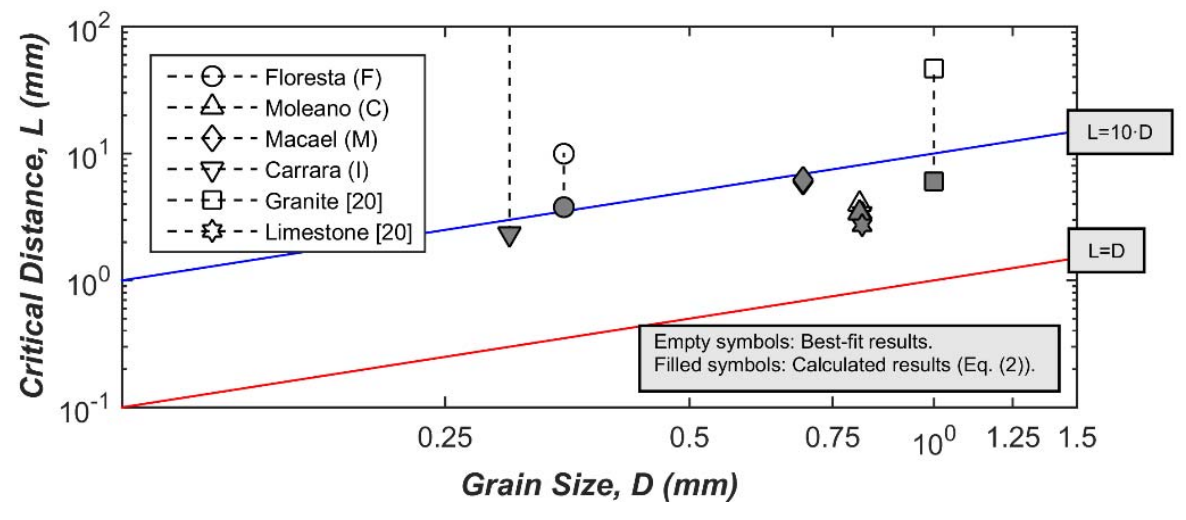

Figure 6. Relation between grain size and L. Rocks studied at [20] have also been included.

\section{CONCLUSIONS}

This paper aims to analyse notch-type defects in four kinds of rocks with different microstructures and characteristics. The study is based on the application of the TCD to the results of an exhaustive experimental program that includes 216 SENB specimens tested in 4-point bending conditions, with notch radii $(\rho)$ varying from $0.15 \mathrm{~mm}$ up to $15 \mathrm{~mm}$.

The obtained values of $L$ allow the load-bearing capacity of any structural component made of these materials to be predicted by the TCD approaches (e.g., PM or LM). In fact, predictions provided by LM have proved to be reasonable even for blunter notches beyond the application range of Eq. (13). Notches with radii smaller than $L$ (which in rocks is of the order of a few $\mathrm{mm}$ ) behave as cracks, which supports the followed procedure, in which similar behaviour for crack and notch-type defects has been assumed for sufficiently limited radii.

Finally, a preliminary microstructural analysis has been performed in order to define the correlation between the critical distance and the grain size of each material. However, further research is required as this relation does not seem to be sufficiently clear. Other possible variables such as grain size density function, porosity or grain composition may also affect the critical distance $L$. 


\section{AKNOWLEDGEMENT}

The authors of this work would like to express their gratitude to the Spanish Ministry of Economy and Competitiveness for financing the National Plan Project (Ref.: BIA2015-67479-R) under the name of 'La Distancia Crítica en la Fractura de Rocas' (The Critical Distance in Rock Fracture).

\section{REFERENCES}

[1] Taylor, D., 2007. The theory of critical distances: a new perspective in fracture mechanics. Elsevier.

[2] Creager, M., Paris, C., 1967. Elastic field equations for blunt cracks with reference to stress corrosion cracking. Int. J. Fract. 3, 247-252.

[3] Glinka, G., Newport, A., 1987. Universal features of elastic notch tip stress fields. Int. J. Fatigue 9, 143150 .

[4] Pluvinage, G., 1998. Fatigue and fracture emanating from notch; the use of the notch stress intensity factor. Nucl. Eng. Des. 185, 173-184.

[5] Timoshenko, S., Goodier, J.N., 1951. Theory of Elasticity. McGraw-Hill, New York.1992.

[6] Madrazo, V., Cicero, S., Carrascal, I.A., 2012. On the point method and the line method notch effect predictions in Al7075-T651. Eng. Fract. Mech. 79, 363-379.

[7] Cicero, S., Madrazo, V., Carrascal, I.A., 2012. Analysis of notch effect in PMMA by using the Theory of Critical Distances. Eng. Fract. Mech. 86, 56-72.

[8] Neuber N., Weiss V., 1962. Trans. ASME paper No. 62-WA-270.

[9] Chen, C.C., Pan, H.I, 1978. In: Chen, C. (Ed.), Collection of Papers on Fracture of Metals (in Chinese). Metallurgy Industry Press, Beijing, pp. 197-219.

[10] Kujawski, D., 1991. Estimation of stress intensity factors for small cracks at notches. Fatigue Fract. Eng. Mater. Struct. 14, 953-965.

[11] Usami, S.I., 1985. In: Tanaka, M.Jono, Komai, K. (Eds.), Current Research on Fatigue Cracks. The Society of Material Science, Kyoto, Japan, p. 119.

[12] Niu, L.S., C.Chehimi, C., G.Pluvinage, G., 1994. Stress field near a large blunted v notch and application of the concept of critical notch stress intensity factor to the fracture of very brittle materials. Eng. Fract. Mech. 49, 325-335.

[13] Bao, Y., Jin, Z., 1993. Size effects and mean strength criterion for ceramics. Fatige Fract. Eng. Mater. Struct. $16,829-835$.

[14] Taylor, D., Merlo, M., Pegley, R., Cavatorta, M.P., 2004. The effect of stress concentrations on the fracture strength of polymethylmethacrylate. Mater. Sci. Eng. A382, 288-294.

[15] Neuber, H., 1958. Theory of notch stresses: principles for exact calculation of strength with reference to structural form and material. Springer Verlag, Berlin.

[16] Peterson, R.E., 1959. Notch sensitivity. In: Sines, G., Waisman, J.L. (Eds.), Metal Fatigue. McGraw Hill, New York, pp. 293-306. 
[17] Lataji, E.Z., 1972. Effect of tensile stress gradient on brittle fracture initiation. Int. J. Rock Mech. Min. Sci. Geomech. Abstr. 9, 569-578.

[18] Ito, T., Hayasi, K., 1991. Physical background to the breakdown pressure in hydraulic fracturing tectonic stress measurements. Int. J. Rock Mech. Min. Sci. Geomech. Abstr. 28, 285-293.

[19] Ito, T., 2008. Effect of pore pressure gradient on fracture initiation in fluid saturated porous media: Rock. Eng. Fract. Mech. 75, 1753-1762.

[20] Cicero, S., García, T., Castro, J., Madrazo, V., Andrés, D., 2014. Analysis of notch effect on the fracture behaviour of granite and limestone: An approach from the Theory of Critical Distances. Eng. Geol. 177, $1-9$.

[21] Anderson, T.L., 2004. Fracture mechanics: fundamentals and applications, Third ed. CRC Press, Florida.

[22] Folk, R.L., 1959, Practical petrographic classification of limestones: Bull. Amer. Assoc. Petrol. Geol. v. $43 / 1$, p. $1-38$.

[23] Dunham, R.J., 1962, Classification of carbonate rocks according to depositional textures: Amer. Assoc. Petrol. Geol. Mem. 1: p. 108-121.

[24] UNE 22950-2 (1990). Mechanical properties of rocks. Strength determination tests. Part 2: Tensile strength. Indirect determination (Brazilian test). AENOR. (In Spanish).

[25] ASTM-PS70:1997, 1997. Provisional test methods for determination of fracture toughness of advanced ceramics at ambient temperatures. American Society for Testing and Materials, Philadelphia.

[26] Ouchterlony, F., 1988. ISRM suggested methods for determining the fracture-toughness of rock. Int. J. Rock Mech. Min. Sci. Geomech. 25, 71-96.

[27] CEN/TS 14425-1:2003, 2003. Advanced technical ceramics-test methods for determination of fracture toughness of monolithic ceramics-part 1: guide to test method selection. Committee for Standarization, European.

[28] Amaral, P.M., Guerra Rosa, L., Cruz Fernandes, J., 2008. Assessment of fracture toughness in ornamental stones. Int. J. Rock Mech. Min. Sci. 45, 554-563.

[29] UNE-EN 13161 (2008). Natural stone test methods - Determination of flexural strength under constant moment. AENOR. (In Spanish).

[30] Srawley, J., Gross, B., 1976. Cracks and fracture. ASTM Spec. Tech. Publ. 601, 559-579.

[31] Taylor, D., 2012. Applications of the theory of critical distances in failure analysis. Eng. Fail. Anal. 18, 543-549.

[32] Taylor, D., 2017. The Theory of Critical Distances: A link to micromechanisms. International Symposium on Notch Fracture. Santander, 29-31 March 2017, pp. 8-13. ISBN: 978-84-617-9463-8. 